\title{
A chemical activity evaluation of two dental calcium silicate-based materials
}

\author{
Renata Chalas ${ }^{1}$, Ewelina Mielko², Joanna Zubrzycka-Wrobel ${ }^{1}$, Jakub NowaK ${ }^{3}$
}

\author{
${ }^{1}$ Department of Conservative Dentistry and Endodontics, Medical University of Lublin, Karmelicka 7, 20-081 Lublin, Poland \\ ${ }^{2}$ Private Dental Practice in Krakow, Poland \\ ${ }^{3}$ Deapartment of Chemistry, The John Paul II Catholic University of Lublin, Poland
}

\begin{tabular}{|c|c|}
\hline ARTICLE INFO & ABSTRACT \\
\hline Received 15 April 2015 & \multirow{3}{*}{$\begin{array}{l}\text { Calcium silicate-based materials are interesting products widely used in dentistry. The } \\
\text { study was designed to compare the chemical reaction between analyzed two preparates } \\
\text { and dentin during cavity lining. In our work, dentinal discs were prepared from human } \\
\text { extracted teeth filled with Biodentine and MTA+. The samples were then analyzed by } \\
\text { way of SEM, EDS and Raman spectroscopy. The obtained results revealed differences } \\
\text { in elemental composition between both materials. Biodentine showed higher activity in } \\
\text { contact with dentine. Moreover, the interfacial layer in the tooth filled by Biodentine was } \\
\text { wider than that in the tooth filled with MTA+. The applied methods of analysis confirmed } \\
\text { that both materials have a bioactive potential which is a promising ability. }\end{array}$} \\
\hline Acc & \\
\hline $\begin{array}{l}\text { Keywords: } \\
\text { cavity lining, } \\
\text { Biodentine, } \\
\text { mineral trioxide aggregate, } \\
\text { MTA+. }\end{array}$ & \\
\hline
\end{tabular}

\section{INTRODUCTION}

In dentistry, cavity lining is a technique based on covering the dentin with an odontotropic material. It protects the pulp from external factors, inhibits the influx of bacteria, intensifies remineralization and induces dental tissue to produce tertiary dentin. All these attributes extend the pulp's vitality. In current dental practice, there are several calcium silicatebased materials showing ion release into dental tissues [6]. That unique feature makes them crucial in biological treatment. Two of these, Biodentine ${ }^{\circledR}$ (Septodont) and MTA+ (Cerkamed), have been favored by dentists. Their similar practical application (f.ex. in direct pulp capping, curing resorptions and covering perforations) and composition make these useful in the healing of the contacted tissues. Indeed, the choice of the material should be governed not only by handling properties, but also by its biocompatibility and sealing, and as well by its clinical success too. Biodentine is similar to MTA (mineral trioxide aggregate) in basic composition. Silicates are the main core of Biodentine. These are a tri-calcium silicate and a di-calcium silicate combination. It also contains carbonate and oxide (as filler), iron oxide (responsible for tint) and zirconium oxide (incorporated as a radiopacifier). Adding calcium chloride to the liquid accelerates the reaction between the components. The

\footnotetext{
* Corresponding author

e-mail: renata.chalas@umlub.pl

tel.: 8152879 20, fax: 815287921
}

reduction of water is provided by a hydrosoluble polymer $[3,9]$. Calcium hydroxide and calcium silicate are the principal components of MTA+. The material also contains calcium phosphate, sodium, potassium and magnesium oxides. The present iron oxide is used for tint, while the contained bismuth allows identification on radiographs. The used liquid is distilled water [4].

The in vitro pilot study was design to compare the composition and chemical reaction between the analyzed two bioactive calcium-silicate based materials and dentine, during cavity lining.

\section{MATERIALS AND METHODS}

Seven extracted human molars (collected in written agreement with every patient) were taken into the study. Using a high rotation hand-piece and diamond bur under water coolant, cavities on the occlusal surfaces were made in every tooth to dentin level. Afterwards, the examined materials were placed into the prepared cavities. Biodentine was prepared by pouring 5 drops of liquid into a capsule and mixing for 30 seconds in a triturator. Through the addition of 2 drops of distilled water and compounding with MTA+ powder by way of mixing with the use of a metal paddle on a glass plate, the material was readied for use. Application was performed at once in all cavities without a conditioning treatment of the dentin. After the completion of the materials' hardening time, every sample was cut perpendicularly, 
into $1.0 \mathrm{~mm}$ thick slices, through the use of a Diamond Cutter Micracut 175, with water rinsing (Fig. 1).

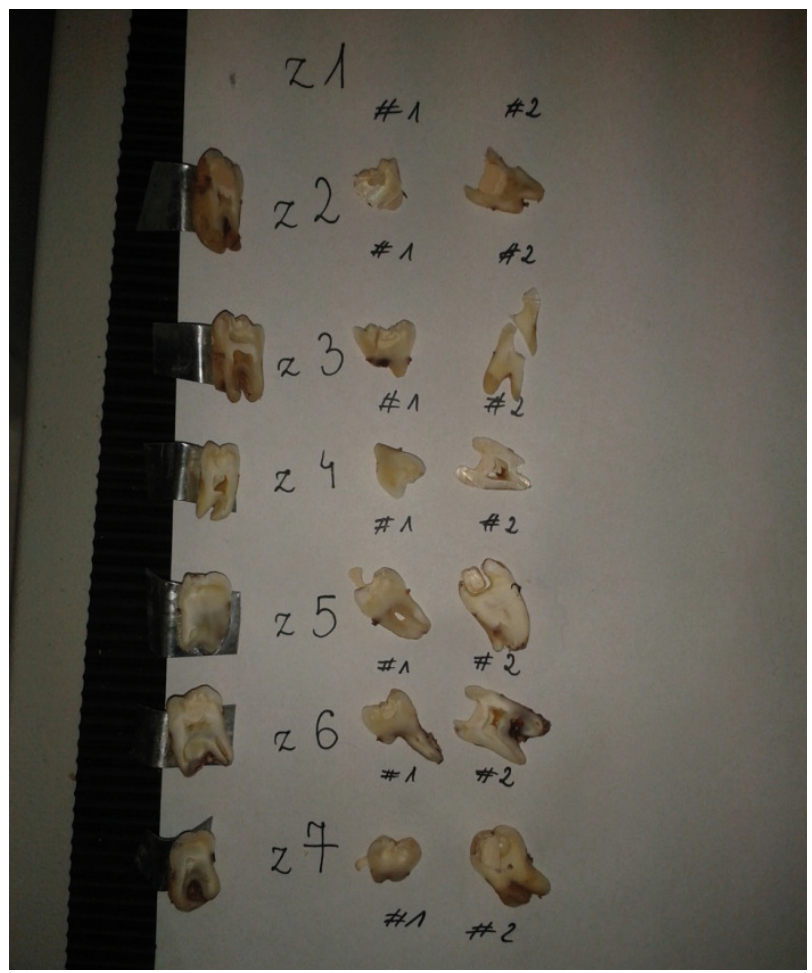

Figure 1. Image of the cut samples

To investigate the effect of Biodentine and MTA+ application on the teeth samples, the following methods were applied: Scanning Electron Microscopy (to reveal information about the morphology and structure of the sample), Energy Dispersive X-Ray Spectroscopy (so as to characterize the elemental composition and chemical characterization of the analyzed volume) and Raman spectroscopy (a spectroscopic technique based on the inelastic scattering of monochromatic light, and used to study the chemical function groups of the samples) $[3,8,13]$.

\section{RESULTS}

The structure of both materials was ascertained and compared by way of SEM images. In these, it was found that both treatments are non-homologous, but Biodentine showed more crystalline formation and better contrast between light and dark crystals. Microscopic analysis of the border between dentin and examined materials revealed a good sealing between Biodentine and dental tissue, in comparison to $\mathrm{MTA}+$. In the case of MTA + , a fragmentary border was visible in the contact with dentin.

The obtained results from the chemical analysis performed through the use of Energy Dispersive X-Ray Spectroscopy, showed significant differences in the composition of both materials. In Figure 2, it can be seen that samples with Biodentine treatment contain definitely more calcium, and a little more oxygen, than that of the MTA+ treatment, while the level of carbon content is comparable (Fig. 2).

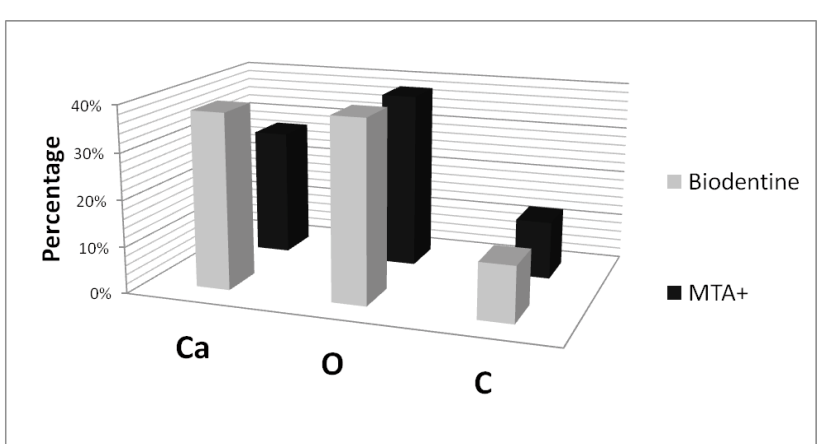

Figure 2. Presence of calcium, oxygen and carbon in Biodentine and MTA+

Through a combined analysis of the SEM images and EDS results, the presence of an interfacial layer was also revealed. This was identified by assessing the level of calcium and silicon ions. In the sample treatments, the amount of silicon ions was significantly higher in the Biodentine layer. What is more, measurement of the width of the transition zone demonstrated that this, in this study, was considerably wider for Biodentine treatment $(53 \mu \mathrm{m})$, than that for MTA+ treatment $(18 \mu \mathrm{m})$.

Raman Spectroscopy enabled the investigators to provide the chemical spectra characterization of dentin, Biodentine and MTA+ treatments. The obtained peak results revealed that the sampled dentin contained apatite, Biodentine contained tricalcium silicate, and both materials contained calcite (Fig.3).

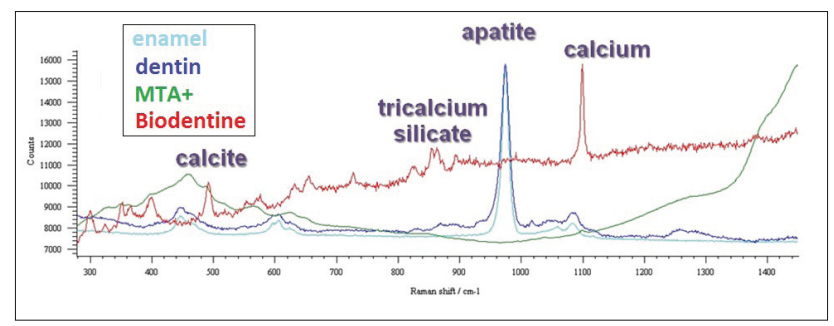

Figure 3. Chemical characterization of spectra for dentin, Biodentine and MTA+, by way of Raman spectroscopy

\section{DISSCUSSION}

The success of any biological treatment greatly depends on the adherence of the filling material to dental tissue. Indeed, the quality of the interface is the key factor for the durability and effectiveness of the material [1]. Furthermore, it is important the applied material used in biological treatment, when come into contact with dental tissues, should not show any toxic, irrigating, inflammatory, allergic, genotoxic or carcinogenic action [12]. The performed research showed minimal micro-leakage values for Biodentine. Its modified composition and a novel way of preparing (prepared within a closed capsule using a mixing device) improved its properties, making the use of Biodentine more convenient and efficient [2]. Such results were also confirmed in the studies of Kokate et al., wherein the marginal seal between Portland cement based materials like Biodentine and MTA was evaluated [7]. The work of Sulaiman et al. [13] also showed that Biodentine had a superior marginal integrity with dental tissue. In such work, the worst results were that of MTA+ treatment when in contact with dentin. This was indicated 
from the examined properties of the material, and reinforced through recommendations in practical clinical situations, in treatment situations where contact is made with the pulp. In the performed study, MTA+ was used experimentally so as to investigate its abilities in contact with dentin.

Several methods for the determination of the chemical activity of dental materials have been recently recommended $[3,4,7,13]$. SEM and EDS (used in the present study) are deemed adequate for showing that calcium and silicon ions were incorporated into the layer between examined materials and dentin. This significant information suggests that both Biodentine and MTA + are bioactive materials. However, the width of the Biodentine transition zone was definitely greater than that of the MTA+ transition zone. This result is in agreement with that obtained by Han et al. in their analysis of the materials' elemental composition and interfacial effects with dentin, which was done by way of using a wavelength-dispersive X-ray spectroscopy electron probe microanalyser with image observation function [5]. Beyond this, the advanced technology of the Raman spectroscope applied in our experiment allowed us to affirm this observation. By utilizing this technique, the higher intensity of calcium ions was clearly visible in the transition zone for Biodentine. Such form of spectral analysis was also employed by Nandini et al. in their examination of the formation of calcium salts in the interface when the mineral trioxide aggregate was covered by a glass-ionomer [10].

The bio-inductive properties of MTA+ and Biodentine are also important from the clinical point of view. Nowicka et al. [11] noticed the presence of a dental bridge and an absence of inflammatory pulp response after direct pulp capping. Moreover, layers of well-arranged odontoblast and odontoblast-like cells were found to form tubular dentin under the osteodentin. Zhirong et al. [14] also confirmed prior results suggesting that Biodentine is a bioactive and biocompatible material which can positively influence healing by enhancing the proliferation, migration and adhesion of human dental pulp stem cells.

\section{CONCLUSION}

The comparative evaluation of the results reveal that Biodentine shows better holding in adhesion to dentin than does MTA+. In addition, the applied methods of analysis showed that both materials have a bioactive potential which is a promising ability. However, regarding their full mechanisms of action, this requires further research.

\section{REFERENCES}

1. Bhat S.S. et al.: Direct pulp capping in an immature incisor using a new bioactive material. Contemp. Clin. Dent., 5, 393, 2014.

2. Cantekin K., Avci S.: Evaluation of shear bond strength of two resinbased composites and glass ionomer cement to pure tricalcium silicate-based cement (Biodentine). J. Appl. Oral Sci., 22, 302, 2014.

3. Chałas R. et al.: Assessment of dentin reaction after Biodentine application. Curr. Issues Pharm. Med. Sci., 26, 435, 2013.

4. Guven Y. et al.: X-ray diffraction analysis of MTA-Plus, MTAAngelus and Dia-Root BioAggregate. Eur. J. Dent., 8, 211, 2014.

5. Han L., Okiji T.: Bioactivity evaluation of three calcium silicatebased endodontic materials. Int. Endod. J., 46, 808, 2013.

6. Khedmat S. et al.: In vitro cytotoxicity of four calcium silicate-based endodontic cements on human monocytes, a colorimetric MTT assey. Restor. Dent. Endod., 39, 149, 2014.

7. Kokate S.R., Pawar A.M.: An in vitro comparative stereomicroscopic evaluation of marginal seal between MTA, glass insomer cement \& biodentine as root end filling materials using $1 \%$ methylene blue as tracer. Endodontology, 24, 36, 2012.

8. Lyon L.A. et al.: Raman Spectroscopy. Anal. Chem., 70, 341R, 1998.

9. Malkondu Ö., Kazandağ M.K., Kazazoğlu E.: A review on Biodentine, a contemporary dentine replacement and repair material. Biomed. Res. Int. 2014, doi: 10.1155/2014/160951.

10. Nandini S., Ballal S.: Influence of glass-ionomer cement on the interface and setting reaction of mineral trioxide aggregate when used as a furcal repair material using laser Raman spectroscopic analysis. J. Endod., 33, 167, 2007.

11. Nowicka A. et al.: Response of human dental pulp capped with biodentine and mineral trioxide aggregate. J. Endod., 39, 743, 2013.

12. Poggio C. et al.: Biocompatibility of a new pulp capping cement. Ann. Stomatol. (Roma), 2, 69, 2014.

13. Sulaiman J.M.A., Yahya M.M., Al-Ashou W.M.O.: An in-vitro scan electron microscope comparative study of dentine-Biodentine interface. J Bagh College Dentistry, 26, 42, 2014.

14. Zhirong L. et al.: Effect of Biodentine ${ }^{\mathrm{mat}}$ on the proliferation, migration and adhesion of human dental pulp stem cells. J. Dent., 42, 490, 2014. 